I.

Aus der II. medicinischen Klinik der Königl. Charité, Berlin (Director: Geh. Med.-Rat Prof. Dr. Fr. Kraus).

\title{
Die Orte der Reizbildung und Reizleitung im menschlichen Herzen.
}

\author{
Von \\ Stabsarzt Dr. Walter Koch. \\ (Hierzu Tafel I.)
}

Die neuesten physiologischen Untersuchungen über die Wertigkeit der einzelnen Herzabschnitte, insonderheit der in ihnen sich findenden Muskelsysieme specifischer Structur, die von uns als cardiomotorische Centren bezeichnet wurden, haben mit den anatomischen Befunden am Herzen eine so weitgehende Uebereinstimmung gezeitigt, dass wir auf Grund dieser Harmonie uns zurzeit ein ganz anderes Bild von der Natur des Herzschlages am gesunden und kranken Herzen machen können, als es noch vor wenigen Jahren möglich erschien. An der Hand des dieser Arbeit beigegebenen Schemas möchte ich in Kürze den augenblicklichen Stand der anatomischen Untersuchungen erläutern und die physiologischen Ergebnissə, soweit sie sich mit ihm in Einklang bringen lassen, zusammenfassen.

Die Abbildung gibt ein Schema der vier Herzhöhlen. Der rechte Vorhof ist halbschematisch gedacht und nur zum Teil eröftnet gezeichnet. Der grün gezeichnete Sinusknoten liegt, wie ersichtlich, an der Einmündungsstelle der Vena cava superior in den rechten Vorhof in der Furche, welche sich zwischen dem mehr häutigen, den Hohlvenen zuzurechnenden Abschnitt des rechten Vorhofes und den an der Taenia terminalis entspringenden Herzohr- bzw. Vorhofstrabekeln (Mm. pectinati) von dem Herzohr-Cavawinkel auf die Cava inferior hinzieht und als Sulcus des Cavatrichters bezeichnet wurde. Er beginnt etwa am HerzohrCavawinkel, zuweilen kurz unterhalb desselben, zuweilen noch dünne Ausläufer eine kleine Strecke weit nach oben an den Cavatrichter aussendend. Unterhalb des Herzohr-Cavawinkels schwillt er bald zu dem von mir als Kopfteil bezeichneten Abschnitte an, verjüngt sich dann zuerst allmählich, dann schneller zu Stamm und unterem Ausläufer und hört etwa in der Mitte $z$ wischen Cava sup. und Cava inf., zuweilen aber noch über die Mitte nach unten reichend, dort auf, wo der sogen. Wenckebachsche Muskelzug (ein ziemlich constantes, etwas stärker hervortretendes Muskelbündel gewöhnlicher Vorhofsmusculatur an der hinteren und seitlichen Wand des Cavatrichters bzw. Vorhofes) den Sulcus kreuzt, wodurch er an dieser Stelle von seiner sonst subpericardialen lage mehr endocardwärts zu liegen kommt. Seine, wie in dem Schema 
angedeutet, netzförmigen Muskelfasern stehen mit der Musculatur des Cavatrichters, des Herzohres und des trabeculären Vorhofsabschnittes allenthalben in Verbindung. Ein directer constant nachweisbarer Zusammenhang durch specifische Musculatur mit dem weiter unten liegenden Asch off-T awaraschen Knoten besteht nicht, sondern die beiden Systeme sind musculös nur durch gewöhnliche Musculatur verbunden.

In dem septalen Abschnitt des rechten Vorhofes und zwar in einem Felde, welches unten von der Ansatzlinie der Tricuspidalis, hinten von der Coronarvene, oben vom Sinusstreifen und vorn von der Pars membranacea begrenzt wird, liegt der erste Abschnitt des sogen. Reizleitungssystems, der Aschoff-Tawarasche oder Atrioventricularknoten. Wie durch die verschiedene Färbung angedeutet, besteht derselbe anatomisch aus zwei mehr oder weniger deutlich trennbaren Abschnitten. Der blau gehaltene Anteil ist der sogen. Vorhofsteil des Aschoff-Tawaraschen Knotens, der auch als Coronarsinusknoten oder kurzweg als Vorhofsknoten bezeichnet worden ist. Da derselbe, wie wir später sehen werden, anatomisch und functionell dem Vorhof zuzurechnen und die Coronarvene als solche ein mehr secundäres Gebilde der Herzen höherer Wirbeltiere ist, möchte ich, damit nicht durch verschiedenartige Bezeichnung Verwechselung und Verwirrung entsteht, den letzten Ausdruck Vorhofsknoten vorschlagen, wobei aber immer zu bemerken ist, dass damit nur der im Schema blau gezeichnete Abschnitt des Atrioventricularknotens gemeint ist. Dieser Vorhofsknoten setzt sich zusammen aus mehr oder weniger parallel bzw. fächerförmig gewellt verlaufenden Muskelfasern, die nach der Mündung der Coronarvene, dem sogen. Coronarvenentrichter, ausstrahlen und sich bis an die Venenmündung verfolgen lassen, die aber auch zum Teil mehr nach oben zur vorderen Umrandung der Fossa ovalis sich hinziehen, und zweitens aus einem mehr geflechtartigen eigentlichen Knotenabschnitt, der in den folgenden Abschnitt des Aschoff-Tawaraschen Knotens, den Kammerteil oder Kammerknoten übergeht.

Dieser im Schema rot gezeichnete Abschnitt des Atrioventricularknotens ist durchweg geflechtartig gebaut und lässt sich beim Huftier durch Glykogenfärbung mit ziemlich scharfer Grenze von dem Vorhofsknoten abtrennen. Der Kammerknoten liegt in dem Winkel, welcher vom Ansatz der T'ricuspidalis und dem vorderen Ende des Sinusstreifens gebildet wird, wo dieser auf die Pars membranacea auftrifft. Der Kammerknoten liegt dabei dem Annulus fibrosus an der Ansatzstelle des vorderen Mitralissegels an und kann sich noch eine Strecke weit bis in die Pars membranacea, die sich in den Annulus fortsetzt, hineinziehen. Eine fixe Grenze zu den Ausläufern des Kammerknotens, dem Hisschen Bündel bzw. Stamm und Schenkeln des Reizleitungssystems, kann wegen individueller Schwankungen nicht gegeben werden. Dass der Stamm des Reizleitungssystems als Fortsetzung des Kammerknotens unter der Pars membranacea auf die obere Kante des Ventrikelseptums stösst, sich dort in die beiden Schenkel teilt, die am Septum entlang zu den Fusspunkten der Papillarmuskeln ziehen und sich in feinen Verästelungen durch die Trabekel zur gesamten Ventrikeltriebmusculatur begeben, ist 
wohl zur Genüge bekannt und oft genug beschrieben. Dass die Fasern des Reizleitungsssystems beim Huftier constant, aber auch oft genug noch beim menschlichen und den Herzen der Säugetiere sich der sogen. falschen Sehnenfäden des Herzens als Weg bedienen, haben wir im Schema im linken Ventrikel angedeutet und dem falschen Sehnenfaden eine öfters beim Menschen beobachtete Richtung gegeben. Die Ausläufer des Vorhofsknotens stehen mit der Musculatur des Vorhofes in fliessender allseitiger Verbindung wie die Ausläufer des Kammerknotens (die Ausbreitungen der Schenkel) mit der Ventrikelmusculatur.

Wir haben also kurz zusammengefasst im Herzen drei anatomisch erkennbare Systeme specifischer Natur:

1. Den Sinusknoten mit Ausläufern (grün),

2. den Vorhofsknoten mit Ausläufern (blau),

3. den Kammerknoten mit Ausläufern (rot).

Diese specifischen Muskelsysteme finden wir, wie bekannt, nicht nur constant bei allen Säugetieren und Menschenherzen, sondern sie lassen sich phylogenetisch bei allen Wirbeltieren bis zu den Fischen in mehr oder weniger prägnanter Weise nachweisen.

Von besonderer Bedeutung erscheint mir aber ihre typische Localisation zu sein, worauf wir früher schon mehrfach hingewiesen haben. Wir finden die specifische Musculatur nach A. Keith und J. Mackenzie an den Grenzbezirken der primitiven Herzabschnitte und zwar im Uebergangsbezirk vom Sinus zum Vorhof und vom Vorhof zur Kammer; an ersterer Stelle den Sinusknoten, an der zweiten das Reizleitungssystem. An diesen Uebergangsstellen liegen aber auch die den Blutstrom regelnden Klappenapparate des Herzens, zu denen die specifischen Muskelsysteme in enger Beziehung stehen. Wir haben deshalb die zugehörigen venösen Klappen des Herzens in das Schema (schwarz) mit eingezeichnet. Da der Sinus venosus ausschliesslich in den rechten Vorhof einbezogen wird, können wir am Säugetierherzen dio Reste der Sinusklappen auch nur in seinem Bereiche uns reconstruieren. Nach Keith und Aschoff entspricht die Lage der rechten Sinusklappe im wesentlichen dem Verlaufe der sogen. Taenia terminalis, der musculösen Leiste des rechten Vorhofes, von welcher die Mm. pectinati entspringen und die in ihrem Hauptteile dem Sulcus des Cavatrichters entspricht. In diesem liegt aber der Sinusknoten, der noch im oberen Drittel oder der oberen Hälfte der rechten Sinusklappe als Rest der ursprünglichen Klappenwinkelmusculatur erhalten ist. Die unteren Ausläufer der rechten Sinusklappe werden durch die Valvula Eustachii und Valvula Thebesii gebildet, in deren gemeinsame in den Sinusstreifen auslaufende Zone auch das untere Ende der linken Sinusklappe einstrahlend zu denken ist, welche die Fossa ovalis hinten umkreisend mit der rechten Sinusklappe oben am sogen. Aortenwinkel, an der Ursprungsstelle der Taenia terminalis zusammentrifft. Wenn somit der Sinusknoten sichere Beziehungen zu den Sinusklappen hat und $z$ war seiner Lage nach vorwiegend im Bereiche der Vena cava superior und der benachbarten Lungenvenen, d. h. im oberen Sinusklappenwinkel, so sind für den Vorhofsknoten die Beziehungen zum 
unteren Sinusklappenwinkel und damit zur Cava inferior und Coronarvene deutlich. Es hat deshalb eine gewisse Berechtigung, wenn man annimmt, dass Sinusknoten und Vorhofsknoten, die sich auch histologisch ähneln, als Reste des ursprünglichen Sinusklappenringes aufgefasst werden.

Die Beziehungen des Kammerknotens und seiner Ausläufer, die sich zuerst zum Klappenstellwerk, den Papillarmuskeln, begeben, sind ebenfalls klar zu Tage liegend. Wenn auch die Verhältnisse nicht mehr so übersichtlich sind, wie beim primitiven Herzen, und die Beziehungen zu den Klappen im höher entwickelten Herzen beim Reizleitungssystem eigentlich nur noch indirecte (durch die Papillarmuskeln) sind, so glaube ich doch, dass man diesen Befunden einen gewissen Wert beilegen muss, da man durch sie dem Verständnis für die Function der specifischen Muskelsysteme näherkommt.

Für die später $z u$ erörternde Frage über die Bewertung dieser Muskelsysteme als Centren des Herzens ist von Wichtigkeit ein Einwand, der gegen die Bedeutung der specifischen Muskelsysteme gemacht werden könnte und der meines Wissens noch nicht ausgesprochen ist. Wie die anatomische Schilderung und Skizze zeigen, liegt nämlich der Sinusknoten eigentlich nicht am Anfange des Herzschlauches, sondern erst an der Grenze vom ersten und zweiten $A$ bschnitt, d. h. zwischen Sinus venosus und Vorhof. Es ist ja auch nicht, wie oft fälschlich angenommen, der Sinusknoten der Rest des Sinus venosus, sondern der Rest der Uebergangsmusculatur, der Klappenwinkelmusculatur zwischen Sinus und Vorhof, ebenso wie das Reizleitungssystem nicht als Vorhof, sondern als Verbindungsmusculatur zwischen Atrien und Ventrikeln anzusprechen ist. Da ein noch höher, d. h. stromaufwärts an den Venen gelegenes Centrum nicht bekannt und nach meinen Untersuchungen auch unwahrscheinlich, der Sinus venosus aber nach allen physiologischen Beobachtungen das Primum movens des Herzens ist, steht die Erklärung der Beziehung des Sinusknotens zum Sinus venosus noch aus; d. h. es ist noch nicht erwiesen, ob beim primitiven Herzen der Sinus selbständig schlägt oder unter dem Einfluss der sino-auriculären Verbindungsmusculatur. Da das physiologische kixperiment bekanntlich zeigt, dass nach Abbindung des Sinus vom Vorhof der Sinus weiterschlagen kann, muss das Erregungscentrum aberhalb der Ligatur liegen. Es fragt sich nur, ob bei diesen Versuchen tatsächlich die Uebergangsmusculatur vollständig anatomisch vom Sinus getrennt ist oder nicht. Nach Angabe der Physiologen (Engelmann, Nicolai) soll der Versuch besser gelingen, wenn man die Unterbindung (bzw. Durchschneidung) etwas unterhalb der Grenzfurche anlegt. Das würde dafür sprechen, dass die Uebergangsmusculatur für das Fortschlagen des Sinus nicht ohne Bedeutung ist. Dass aber auch nur geringe Reste von specifischer Musculatur immer noch als Centren wirken können, ist bekannt.

Dass beim Säugetier und Menschen die Verhältnisse infolge völliger Einbeziehung des Sinus venosus in den rechten Vorhof andere sind als beim niederen Tiere, ist nur bis zu einem gewissen Grade zuzugeben, da der Grundtypus des Contractionsablaufes und infolgedessen auch wohl 
die dazu gehörige Reizbildung für den complicierter gebildeten Herzschlauch höherer Wirbeltiere derselbe sein muss. Das, was als Rest der eigentlichen Sinusmusculatur beim Menschen- und Säugetierherzen anzusprechen wäre, liegt ebenfalls venenwärts von den im Schema angedeuteten Sinusklappen und damit stromaufwärts vom Sinusknoten. Ich möchte mich aber im gewissen Sinne der Anschauung J. Mackenzies anschliessen, dass wir am höheren Wirbeltierherzen in der Musculatur, welche stromaufwärts von der Taenia terminalis liegt, also vorwiegend der beiden Cavatrichter, in Wirklichkeit keine eigentlichen Sinusmusculaturreste vor uns haben, sondern dass es sich da um gewöhnliche, nachträglich durch die Einstülpung des Sinus herübergeschobene Vorhofsmusculatur handelt, die sich ja auch histologisch in nichts von der übrigen Musculatur unterscheidet. Es sind daher wirkliche histologisch nachweisbare Sinusreste am Säugetier- und Menschenherzen nicht mehr nachzuweisen, sondern nur noch die Uebergangsfasern in Gestalt des Sinusknotens. Wir können aber für das Säugetierherz nach aller Erfahrung der letzten Jahre wohl ziemlich sicher den Sinusknoten (und nicht die stromaufwärts gelegene Cavatrichtermusculatur) als Schrittmacher des Herzens ansehen. Wenn diese Tatsache auch gut begründet erscheint, darf man doch nur mit gewisser Reserve vom Säugetierherzen auf das primitive Wirbeltierherz zurückschliessen, und ich glaube, wie gesagt, dass man ohne physiologisches Experiment, welches anatomisch controlliert werden müsste, diese Frage nicht entscheiden kann.

Ich komme nunmehr zu den physiologischen Ergebnissen, welche in Hinblick auf das beigegebene Schema kurz zusammengestellt seien, soweit sie auf Grund der Uebereinstimmung mit den anatomischen Daten uns ein einigermassen anschauliches Bild von den Vorgängen bei dem Rhythmus der Herzbewegung geben können. Ich stütze mich dabei im wesentlichen auf die Untersuchungen von Ganter und Zahn, welche die verschiedenen cardiomotorischen Centren bis zu den Ausläufern des Kammerknotens hin experimentell erforscht haben. Danach wird die rhythmische Herzaction in der Norm vom Sinusknoten beherrscht. Er verhält sich als erster bei der Herzaction elektronegativ gegenüber allen anderen Herzabschnitten, und sein Kopfteil wieder steht functionell über seinen anderen Abschnitten (Wybauw Lewis). Von ihm aus kann durch Erwärmung und Abkühlung die Frequenz des Herzschlages willkürlich positiv oder negativ beeinflusst werden, eine Wirkung, die von anderen beliebigen Vorhofsstellen nicht oder doch längst nicht in gleich ausgezeichneter Weise hervorgerufen werden kann (Ganter und Zahn, Brandenburg und Hoffmann). Wird der Sinusknoten anatomisch oder functionell ausgeschaltet, so können die stromabwärts gelegenen Centren für ihn eintreten, und zwar sowohl der Vorhofsknoten wie der Kammerknoten und seltener dessen Ausläufer. Unter welchen Bedingungen die verschiedenen Abschnitte in Function treten, ist noch nicht völlig klar. Jedenfalls ist es meistens der Vorhofs- oder Kammerteil des Atrioventricularknotens, seltener, wie gesagt, die Kammerabschnitte des Reizleitungssystems. Hat der Vorhofsknoten die Führung übernommen, der für gewöhnlich dem Sinusknoten untergeordnet ist, da er 
nur nach Ausschaltung desselben seine führende Tätigkeit geltend macht, so erkennt man das an der geringeren Frequenz des Herzschlages, an der Verkürzung des $A_{s}-V_{s}$-Intervalls (welches aber noch positiv bleibt) und an dem Negativwerden der Vorhofszacke im Elektrocardiogramm (Lohmann, Hering, Ganter und Zahn, Brandenburg und Hoffmann). Aber auch der Kammerknoten kann gleich für den Sinusknoten einspringen, wobei die Herzaction eine wesentlich niedrigere Frequenz aufzuweisen pflegt, das $A_{S}-V_{S}$-Intervall aufgehoben wird (so dass Vorhof und Kammer synchron schlagen) und die Vorhofszacke des Elektrocardiogramms verschwindet bzw. überlagert ist.

Es ist bis jetzt noch nicht gelungen, die Stelle sicher festzulegen, an welcher das $A_{S}-V_{S}$-Intervall gleich Null wird. Die Verkürzung desselben tritt allmählich ein, je tiefer man die Centren absucht. Theoretisch könnte man an die Grenze zwischen Vorhofs- und Kammerknoten denken und annehmen, dass der Vorhof noch so lange rom Vorhofscentrum aus betätigt wird, wie das $A_{S}-V_{S}$ - Intervall Werte über Null hat und dass er rückläufig rom Kammerknoten seine Reizimpulse empfängt, also von einem nicht mehr zum Vorhof gehörigen Abschnitte der cardiomotorischen Centren, wenn das $A_{S}-V_{S}$-Intervall aufgehoben ist. An dieser Stelle etwa müsste daher auch die bekannte Ueberleitungsverzögerung stattfinden, deren Sitz in dem complicierten Flechtwerk des Kammerknotens gesucht wurde (Hering, Zahn, Koch), die man aber auf Grund theoretischer Ueberlegung auch in der Uebergangszone vom Vorhofs- zum Kammerknoten vermuten kann (Aschoff), da ja bei der Tätigkeit des letzteren der Vorbof eigentlich schon rückläufig erregt wird. Jedenfalls ist experimentell erwiesen, dass sich aus der Gesamtmasse des Aschoff-Tawaraschen Knotens, der anatomisch als aus zwei verschiedenen Abschnitten bestehend angesehen werden muss, auch physiologisch functionell verschiedenwertige Zonen unterscheiden lassen.

Das hat sich nun nicht nur bei localen physiologischen Experimenten gezeigt, sondern auch, wie aus einer unlängst erschienenen Arbeit von Ganter und Zahn hervorgeht, bei indirecter Untersuchung durch den Vagus. Es ist schon länger bekannt, dass (wenn auch nicht in allen Fällen, so doch vorzugsweise) der rechte Vagus im wesentlichen auf den Sinusknoten, der linke auf den Aschoff-Tawaraschen Knoten einwirkt (Hering, Lohmann, Rotberger und Winterberg, Cohn, Ganter und Zahn). Dass tatsächlich diese Centren die Angriffsstelle der Vaguswirkung sind, konten die letztgenannten Untersucher dadurch noch beweiskräftiger hervorheben, dass sie durch Erwärmung der betreffenden Centren die Vaguswirkung vermindern, aufheben oder übercompensieren konnten. Was uns hier für die Zweiteilung des A.-V.-Knotens besonders interessiert, ist die Tatsache, dass bei vorübergehender oder definitiver Ausschaltung des Sinusknotens nach Vagusreizung dieser Reiz nicht auf alle Abschnitte des A.-V.-Knotens gleich intensiv zu wirken braucht, so dass der mindergehemmte Teil die Führung behält und die wechselnden $\mathrm{A}_{\mathrm{s}}-\mathrm{V}_{\mathrm{s}}$-Intervallwerte (positiv, null, negativ) anzeigen, welcher Abschnitt am wenigsten bzw. am stärksten betroffen ist. So liess sich durch Vagusreiz nach Ausschaltung des Sinusknotens Ueberleitungsstörung 
zwischen Vorhof und Kammer erzeugen, ähnlich wie bei Coronarsinusrhythmus (Vorhofsknoten) durch Abkühlung der Vorhofsknotenabschnitte.

Als letzte bisher physiologisch (ebenfalls von Ganter und Zahn nachgewiesene) Reizbildungsetappe kommen die Ausläufer des Kammerknotens, d. h. Stamm und Schenkel des Reizleitungssystems in Betracht, wie durch die Methode der localen Abkühlung und Erwärmung nachgewiesen wurde, wobei hervorgehoben werden muss, dass deutliche Wirkung nur von den Stellen der Ventrikel erzielt wurde, welche dem Verlauf der Schenkel entsprachen, dass diesen Stellen zum mindesten ein wesentliches Uebergewicht über die benachbarte Ventrikelmusculatur zugesprochen werden muss. Die Automatie dieser unterhalb des Kammerknotens gelegenen Centren tut sich kund durch ebenfalls beträchtliche Frequenzverminderung des Herzschlages und stets negatives $A_{S}-V_{S}-$ Intervall.

Es ist nicht meine Absicht, im Rahmen dieser Zeilen, die einen kurzen Ueberblick über den gegenwärtigen Stand unserer Anschauungen von der anatomisch-physiologischen Localisation der Herzcentren geben sollen, die Pathologie der Reizbildungscentren eingehender zu besprechen. Nur einige kurze Hinweise auf ihre Beziehungen $\mathrm{zu}$ den chronischen Arhythmien seien mir erlaubt. Ich habe schon an anderer Stelle und zuletzt gelegentlich des internationalen Congresses in London darauf hingewiesen, dass die anatomischen Untersuchungen an Herzen von Fällen von Pulsus irregularis perpetuus eine einheitliche Aetiologie in Bezug auf die specifischen Nuskelsysteme nicht erkennen lassen. Die hauptsächlichsten Befunde an den Herzen solcher Fälle deuten einerseits auf Stauung, die besonders den rechten Vorhof mitbetrifft, andererseits auf chronische entzündliche Processe im Bereiche der Mitral- und Aortenklappen und deren Ansatzgebieten, der Aortenwurzel bzw. der Pars membranacea und des Annulus fibrosus der Scheidewände. Da sich ausserdem in einer Reihe von Fällen eine gewisse Ueberdehnung und regressive Veränderung der specifischen Muskelsysteme, besonders des Sinusknotens, zeigten, glaubte ich die Hypothese aufstellen zu können, dass man beim Pulsus irregularis perpetuus sich den Mechanismus der Herzaktion durch ein Widerspiel zwischen erschöpftem Sinusknoten und für ihn sich einsetzendem Vorhofknoten bewirkt denken könne, und dass das Infunktiontreten des Vorhofknotens bei fehlender oder nicht genügender Betätigung des Sinusknotens eventuell auf chemische, mechanische oder entzündliche Reize zurückgeführt werden könne. Ich hob dabei die venöse Stauung besonders im Coronarvenengebiet, wo der Vorhofsknoten ausläuft, für Fälle von Emphysem, Arteriosklerose und dergleichen hervor, ferner die nahen Beziehungen des Vorhofs- und Kammerknotens zu den Teilen der Herzscheidewände, an welchen die bei Arhythmia perpetua procentualiter so häufig erkrankten Mitral- und Aortenklappen ansetzen, so dass man bei den vorgeschrittenen Fällen, besonders den schweren Mitralstenosen, an directe mechanische Zerrung und Erschütterung dieses wichtigen Gebietes denken muss, abgesehen von den entzündlichen, von den Klappen übergreifenden Processen und der auch bei diesen Herzen bestehenden venösen Stase. Auch die un- 
regelmässige Durchblutung der Herzcentren, die man auf Grund der histologischen Veränderungen der Knotengelässe annehmen muss, sind im Zusammenhange mit der allgemeinen Stauung zu nennen. Dass man in selteneren Fällen (z. B. bei Basedow) auch an indirecte nervöse Einflüsse auf die entsprechenden Centren denken muss, ist nach den vorerwähnten Untersuchungen über die Angriffspunkte der Vagi wohl anzunehmen.

Aber auch auf Grund physiologischer Experimente hat man einige Berechtigung, für eine gewisse Anzahl von Fällen an dominierende Tätigkeit des Vorhofsknotens zu denken. Es ist dem Kliniker bekannt, dass paroxysmale Tachycardie und Arbythmia perpetua wesensverwandte pathologische Processe sein müssen, da das eine Krankheitsbild in das andere bei demselben Patienten übergehen und dieser Wechsel sich häufiger wiederholen kann. Ganter und Zahn erwähnen nun ein Experiment, in welchem sie durch leichte mechanische Reizung der hinteren Vorhofswand Tachycardien mit einer Frequenz von etwa 250 hervorrufen konnten. Da das Vorhofselektrocardiogramm dabei eine negative Zacke aufwies, vermuteten sie im Vorhofsknoten das augenblickliche Centrum, und tatsächlich konnten sie auch durch Abkühlung dieser Stelle die Tachycardie zum Verschwinden bringen. Ferner beobachteten sie, dass Druckdifferenzen bei der Durchspülung künstlich genährter Herzen Wechsel im Ausgangspunkt der Herzreize in der Weise hervorrufen können, dass Druckerniedrigung dem Vorhofsknoten, Druckerhöhung dem Sinusknoten die Führung verschaffen konnte. Diese Beobachtungen bestätigen im gewissen Sinne unsere auf Grund der anatomischen Untersuchungen gegebenen Hypothesen (wenn auch nur mit Vorsicht vom Experiment auf die Verhältnisse am Lebenden geschlossen werden darf), dass für die erwähnten chronischen Arhythmien die Ursprungsstelle der Herzreize unter Umständen durch ein Widerspiel zwischen den anatomisch festgelegten Centren bestimmt wird und dass auch pathologische Reize (Stauung, Erschütterung usw.) für das Zustandekommen der Herzstörung für manche Fälle in Betracht kommen werden. Ich glaube, dass gerade für die chronischen Arhythmien mehr die Reizhypothese in Frage kommt, während man bei den insufficienten reinen Herzhypertrophien (Nephritikerherz) mehr die Erschöpfungshypothese ins Auge fassen müsste.

Anmerkung! Eine ausserordentlich interessante Arbeit über „Das Elektrocardiogramm der Arhythmia perpetua" bringt, wie ich nach Abschluss meiner Zusammenfassung erfahre, K. Fahrenkamp in Bd. 112 des Deutschen Archivs für klinische Medizin. Besonders bemerkenswert erscheinen mir seine Beobachtungen, dass man für den Pulsus irregularis perpetuus die Vorhofsstörung weniger oft in Flimmern als vielmehr in einer Vorhofstachysystolie zu suchen hat. Das stimmt mit den anatomischen Untersuchungen insofern überein, als man tatsächlich oft genug die Musculatur des Vorhofes zwar wohl überdehnt und regressiv, aber doch nicht so hochgradig verändert findet, dass man keine oder nur flimmernde Tätigkeit von ihr voraussetzen könnte. Da aber nicht nür die Beschaffenheit der gewöhnlichen Musculatur, sondern mehr die Func- 
tion der Reizbildungsstellen für die Erklärung der Tachysystolien in Frage kommt, ist es von besonderem Interesse, aus seinen Elektrocardiogrammen über ihre Tätigkeit zu erfahren. Wie F. zeigt, ist das Bild ein ausserordentlich vielgestaltiges und abnorme Erregungen können von allen Teilen der specifischen Muskelsysteme ausgehen, auch von den Kammerabschnitten, wie schon Hoffmann gezeigt hat. Dass auch Ueberleitungsstörungen mit im Spiele sind, liess sich an der Hand der Curven deutlich zeigen und ist ja auch ohne weiteres verständlich, wenn man sich vergegenwärtigt, dass man bei Pulsus irregularis perpetuus bei einer Ventrikelschlagzahl von etwa $50 / 150$ mit Vorhofstachysystolien von 200/400 und darüber, ja mit Flimmern rechnen muss. Ich glaube, dass diese Tatsachen mit unseren Beobachtungen insofern in Einklang gebracht werden können, als wir öfters auch am Reizleitungssystem, wie früher erwähnt, Zeichen regressiver Veränderungen besonders der Ueberdehnung finden konnten. Wie man sich aber im Einzelnen die Ueberleitungsstörungen erklären will - ob die frequent kommenden Vorhofsreize erst in gewisser Summation wirken, ob die Kammerabschnitte des Reizleitungssystems nicht mehr anspruchsfähig genug sind, oder die Ventrikeltriebmusculatur selbst - , ist nach wie vor eine offene Frage, ebenso woher die Frequenz der Vorhofstätigkeit kommt, für welche ich auf die von uns gegebenen Erklärungsmöglichkeiten verweise. Auch F. nimmt für manche Fälle ektopische Vorhofsreize, die sich durch negative Vorhofszache kund tun (und deshalb wohl auf den Vorhofsknoten zu beziehen sind), als die Quelle der Vorhofserregungen an.

Die der Arbeit beigegebene Tabelle gibt im übrigen an klinischen Diagnosen in grossen und ganzen dieselben Herzveränderungen an, wie ich sie pathologisch-anatomisch in annähernd demselben Verhältnis fand und zwar Mitralstenosen in über der Hälfte der Fälle, demnächst Arteriosklerose, Aortenfehler und Lues.

\section{Erklärung der Abbildung auf Tafel I.}

Schematische Darstellung der specifischen Muskelsysteme im menschlichen Herzen (nach Aschoff-Koch).

Rechter Vorbof nur zun Teil eröffnet, die übrigen Herzhöhlen aufdem Durchsehnitt. W. = Wenckebachscher Muskelzug; L.S.K. = Linke Sinusklappe; V.E. = Valvula Eustachii; V.Th. = Valvula Thebesii; S.Str. = Sinusstreifen; V.S.u. K.S. $=$ Vorhofsu. Kammerscheidewand; S.K. (grün)=Sinusknoten; V.K. (blau)=Vorhofsknoten bzw. Vorhofsteil des Aschoff-'Tawaraschen Knotens; Reizleitungssystem (rot); K.K.=Kammerknoten bzw. Kammerteil des Aschoff-Tawaraschen Knotens; St. $=$ Stamm des Reizleitungssystems (Hissches Bündel); R.S.u.L.S. = Rechter und linker Schenkel des Reizleitungssystems; Au.r.u.Au.I. = Ausbreitungen des Reizleitungssystems; f.S. = falscher Sebnenfaden. 


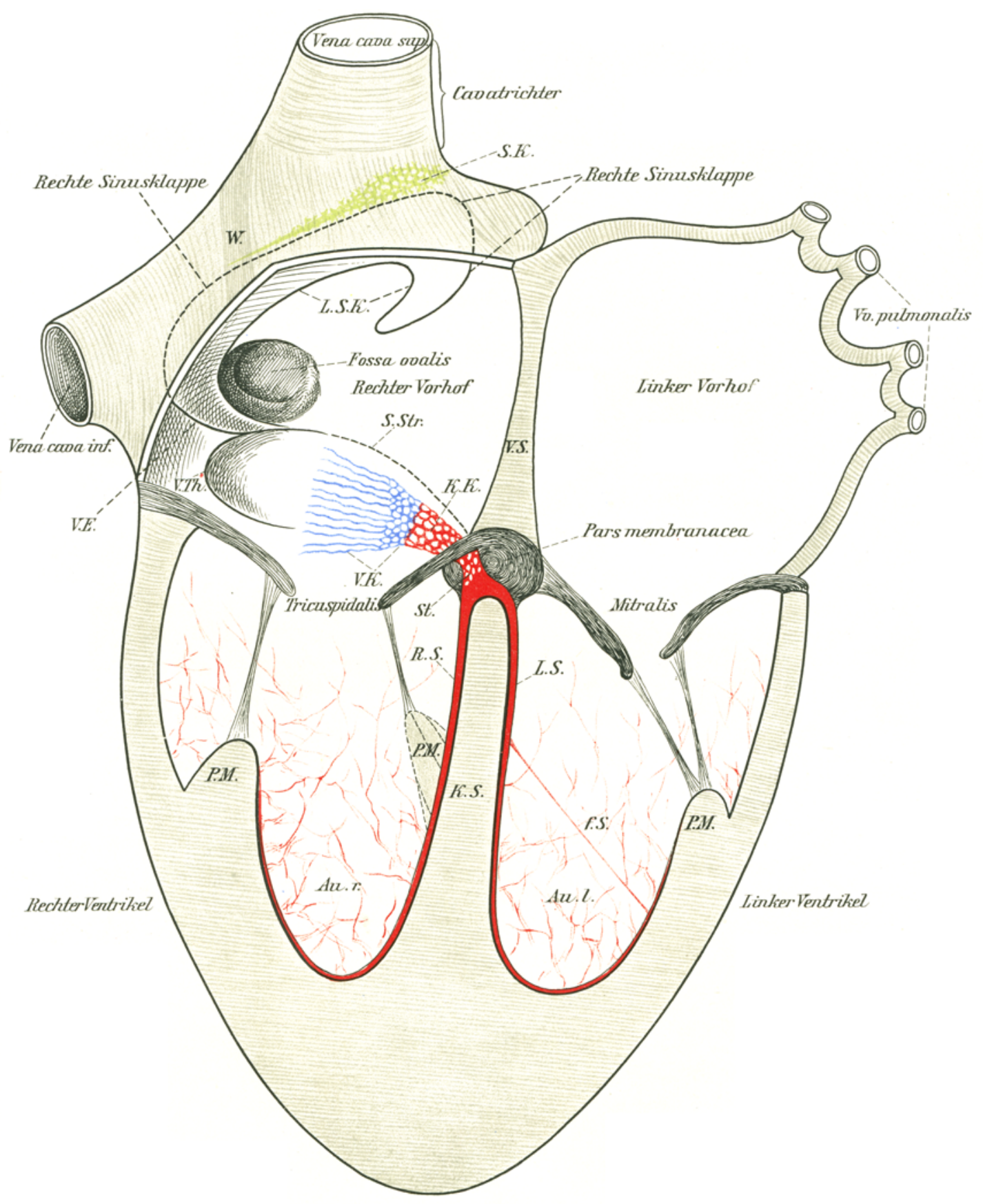

